\title{
Incessant Tachycardia Using a Concealed Atrionodal Bypass Tract
}

\author{
ADAM ZIVIN, M.D., and FRED MORADY, M.D. \\ From the Division of Cardiology, Department of Internal Medicine, \\ University of Michigan Medical Center, Ann Arbor, Michigan
}

\begin{abstract}
Atrionodal Bypass Tract. A case of incessant supraventricular tachycardia continuing despite AV block is reported. Atrial tachycardia and AV nodal reentrant tachycardia were excluded, as was orthodromic tachycardia using a concealed accessory AV pathway. The earliest retrograde atrial activation was at the posterolateral tricuspid annulus, and the tachycardia was eliminated by ablation at this site. The findings in this case are explained only by a concealed atrionodal pathway. (J Cardiovasc Electrophysiol, Vol. 9, pp. 191-195, February 1998)
\end{abstract}

catheter ablation, accessory pathway, supraventricular tachycardia, orthodromic tachycardia

\section{Introduction}

Atrionodal, atrio-Hisian, and atriofascicular pathways with manifest conduction are recognized entities, ${ }^{1-3}$ but a concealed atrionodal pathway participating in a reentrant supraventricular tachycardia has never been reported. The purpose of this case report is to describe a patient who had an incessant supraventricular tachycardia using a concealed atrionodal pathway.

\section{Case Report}

A 39-year-old man was referred for treatment of an incessant, narrow-QRS complex tachycardia of 2-months' duration. The tachycardia was associated with symptoms of palpitations and exercise intolerance. Treatment with digoxin, atenolol, and verapamil had been ineffective. Physical examination demonstrated a blood pressure of 115/60 $\mathrm{mmHg}$, heart rate of 150 beats/min, and no other cardiac abnormalities. The ECG demonstrated a short-RP supraventricular tachycardia at a rate of 150 beats/min. An echocardiogram demonstrated

Address for correspondence: Fred Morady, M.D., Division of Cardiology, Department of Medicine, University of Michigan Medical Center, 1500 E. Medical Center Drive, B1F245, Box 0022. Ann Arbor, MI 48109-0022. Fax: 313-936-7026.

Manuscript received 13 November 1997; Accepted for publication 12 December 1997. no structural abnormalities; the left ventricular ejection fraction was normal.

After informed consent was obtained and 72 hours after discontinuation of all antiarrhythmic drugs, an electrophysiologic procedure was performed. Three multipolar electrode catheters were inserted into a femoral vein and positioned in the high right atrium, His-bundle position, and right ventricular apex. A deflectable, thermistor-tipped 7-French quadripolar electrode catheter that had a 4-mm distal electrode and interelectrode spacing of 2-5-2 $\mathrm{mm}$ was used for mapping and ablation (EP Technologies, Mountain View, CA, USA). Intracardiac electrograms were filtered at 50 to $500 \mathrm{~Hz}$ and recorded along with several ECG leads on a Mingograf-7 analog recorder (Siemens-Elema, Solna, Sweden) at a paper speed of $100 \mathrm{~mm} / \mathrm{sec}$. Pacing was performed at twice diastolic threshold with a programmable stimulator (Bloom Associates Ltd., Reading, PA, USA) using stimuli $2 \mathrm{msec}$ in duration. Radiofrequency energy was delivered at a frequency of $500 \mathrm{kHz}$ (EP Technologies).

The incessant tachycardia was present at the time of the electrophysiologic procedure (Fig. 1). The cycle length of the tachycardia was 355 $\mathrm{msec}$, the atrial-His (AH) interval was $100 \mathrm{msec}$, and the His-ventricular (HV) interval was 40 msec. The pattern of atrial activation was eccentric, with the high right atrial electrogram preceding the atrial electrogram recorded at the His- 


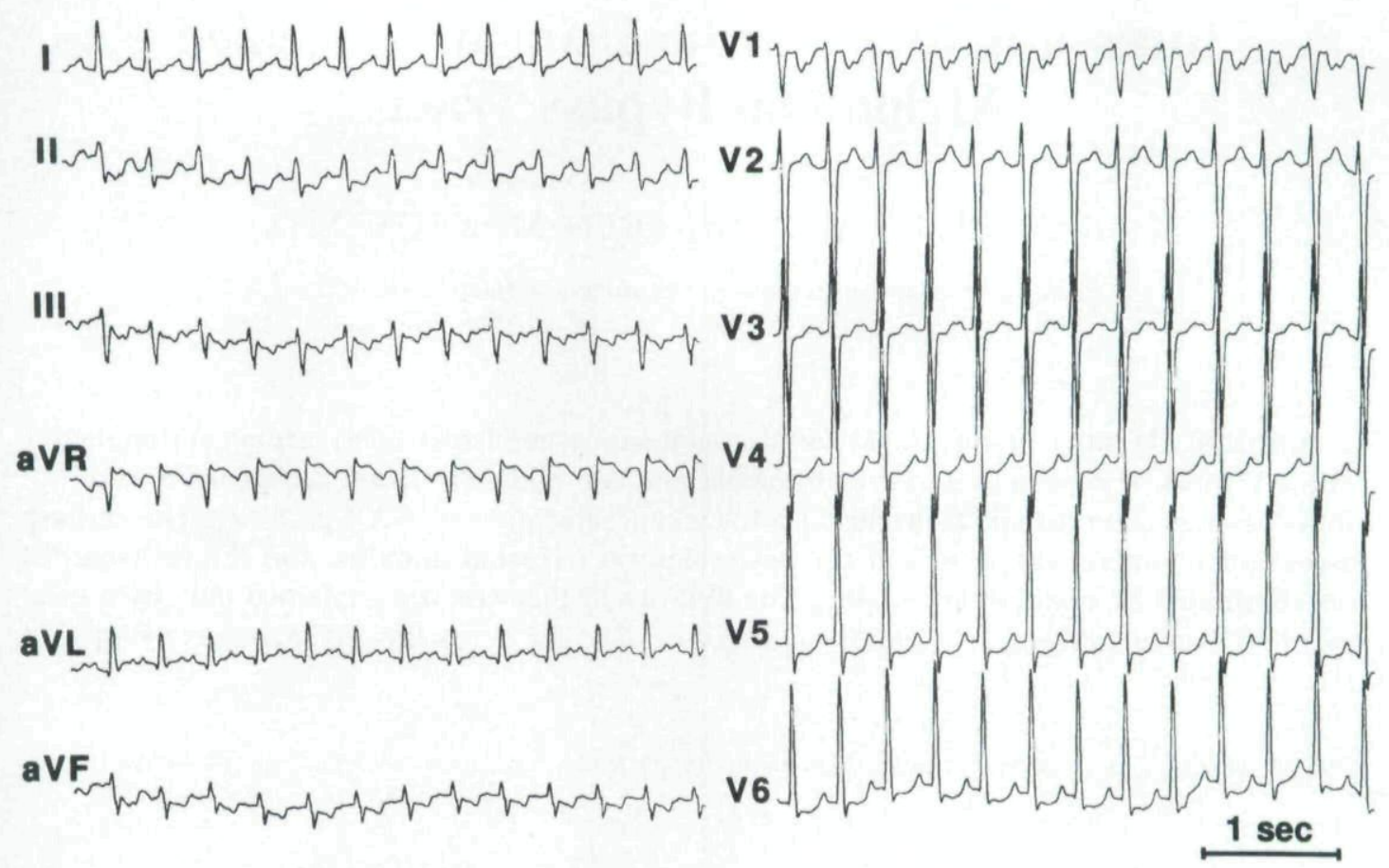

Figure 1. Twelve-lead ECG of a short R-P tachycardia at a rate of 170 beats $/ \mathrm{min}$.

bundle position. The interval from the onset of the QRS complex to the atrial electrogram recorded at the His-bundle position was $150 \mathrm{msec}$. The tachycardia was terminated by rapid atrial or ventricular pacing, but always was reinitiated within 2 to 3 seconds by a premature ventricular depolarization (Fig. 2). The sinus complexes had an AH interval of $90 \mathrm{msec}$ and an $\mathrm{HV}$ interval of $40 \mathrm{msec}$. The VA block cycle length was $280 \mathrm{msec}$.

All spontaneous and pacing-induced terminations of the tachycardia were associated with AV block. Ventricular pacing during the tachycardia or during sinus rhythm at the tachycardia cycle length resulted in the same eccentric pattern of atrial activation as occurred during tachycardia. Introduction of a ventricular extrastimulus at the right ventricular apex during tachycardia when the His bundle was refractory consistently failed to advance or delay the next atrial electrogram (Fig. 3). There was a spontaneous episode of AV nodal block during the tachycardia, with no interruption of the tachycardia (Fig. 4).

Mapping during tachycardia demonstrated that the earliest site of retrograde atrial activation was at the posterolateral tricuspid annulus, at 7 o'clock in the LAO view (Fig. 5). A single 60-second application of radiofrequency energy at this site terminated the tachycardia. No VA conduction was present postablation, and the tachycardia was no longer inducible. The AH and $\mathrm{HV}$ intervals were unchanged after ablation. The patient was not treated with antiarrhythmic medications and has remained free of symptomatic tachycardia during 5 months of follow-up.

\section{Discussion}

\section{Differential Diagnosis}

Based on the ECG, it was felt that the most likely mechanisms of the short-RP tachycardia recorded in this patient were atypical AV nodal reentrant tachycardia, atrial tachycardia, and orthodromic reciprocating tachycardia using a slowly conducting, concealed accessory pathway. Upon recording the intracardiac electrograms, the possibility of AV nodal reentrant tachycardia was immediately ruled out by the eccentric pattern of atrial activation, with the high right atrial electrogram preceding the low right atrial septal electrogram. An atrial tachycardia was quickly excluded by the observation that ventricular pacing resulted in the same eccentric pattern of atrial activation as that recorded during the tachycardia, and by the fact that termination of the tachycardia, either spontaneously or by atrial pacing, was dependent upon the occurrence of AV block. At 


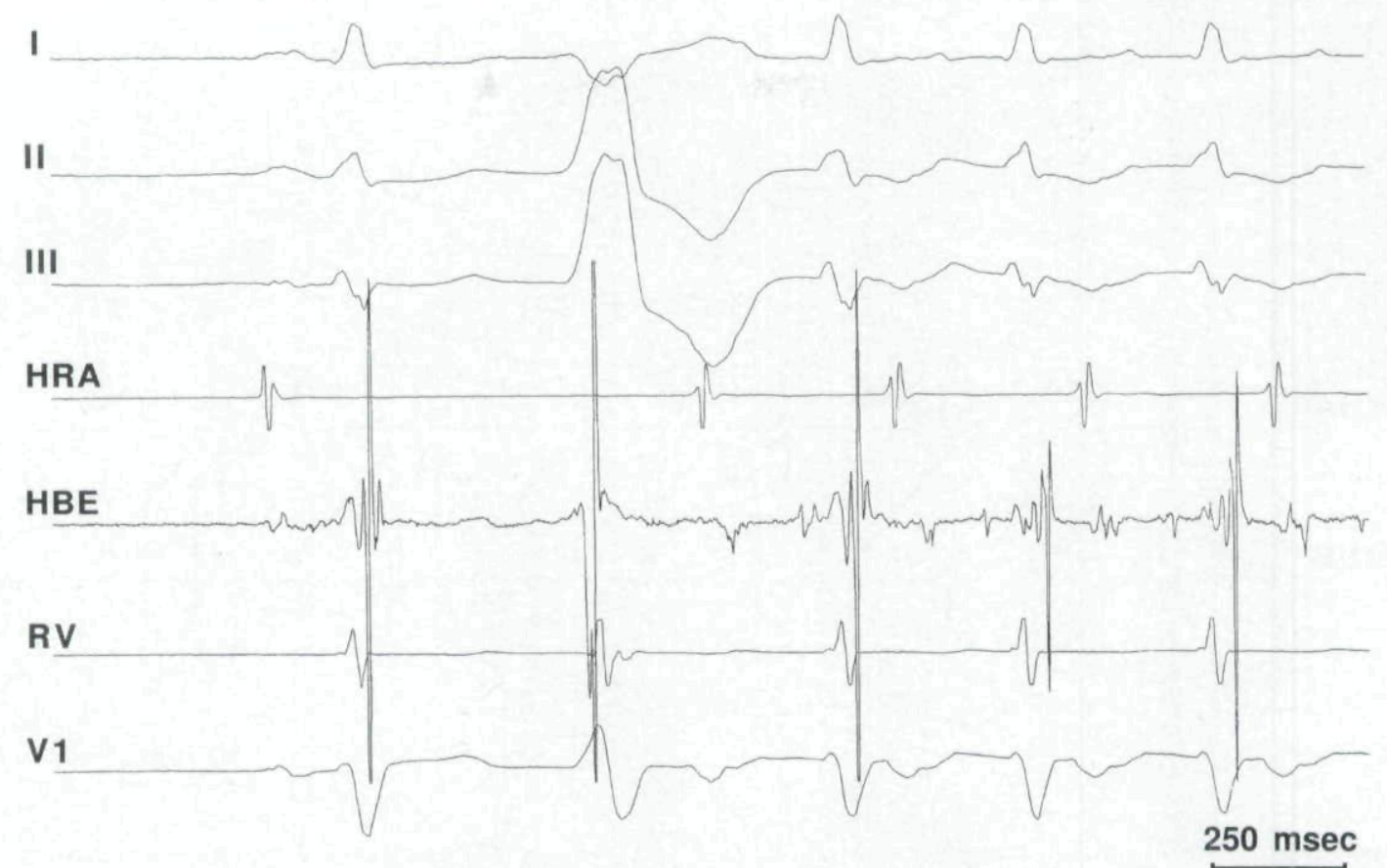

Figure 2. Initiation of the tachycardia by a spontaneous ventricular premature depolarization that results in an eccentric pattern of retrograde atrial activation, similar to the atrial activation pattern recorded during the tachycardia. Shown are leads $I, I$, and III, high right atrial electrogram (HRA), His-bundle electrogram (HBE), right ventricular apex electrogram (RVA), and lead $V_{l}$.

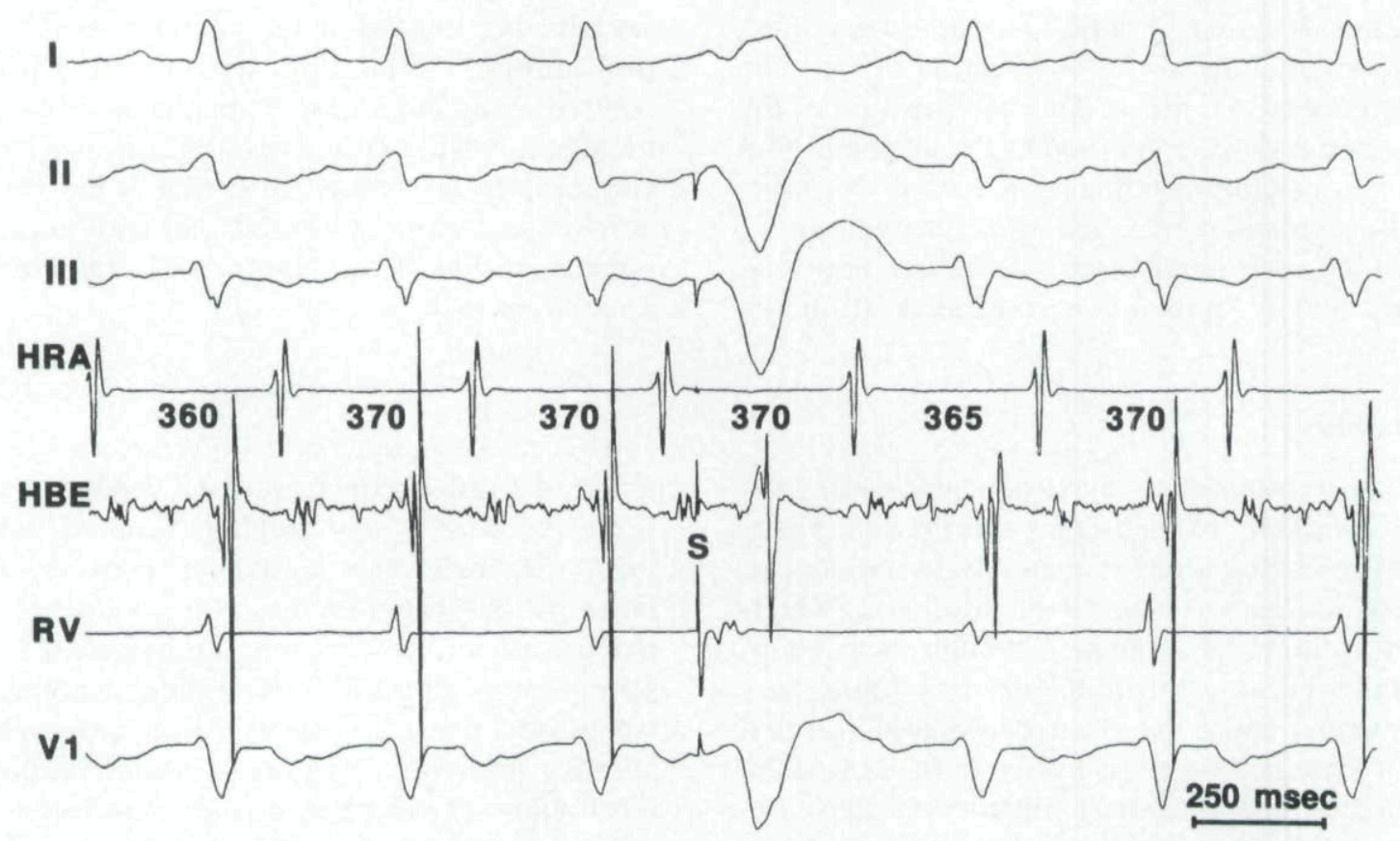

Figure 3. Introduction of a ventricular extrastimulus $(S)$ at the right ventricular apex during His-bundle refractoriness fails to advance or delay the next atrial electrogram. The atrial cycle length varies between 360 and 370 msec and is unaffected by the ventricular extrastimulus. Abbreviations as in Figure 2. 

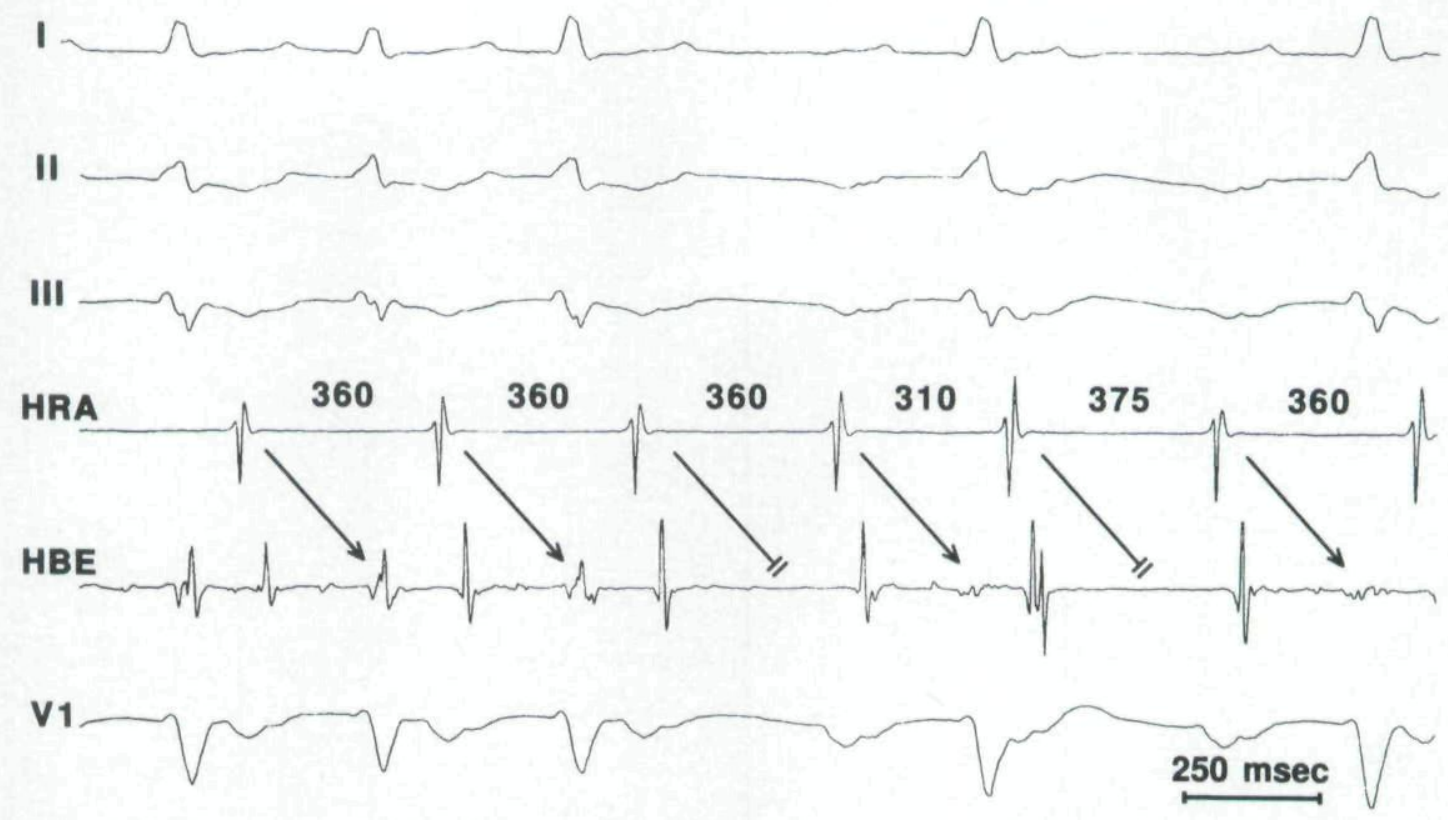

Figure 4. The tachycardia continues despite two instances of AV nodal block. The atrial cycle lengths are as indicated in milliseconds. Note that the atrial cycle length is stable at $360 \mathrm{msec}$ before and during the first instance of AV block. Before the second instance of AV block, the atrial cycle length shortens to $310 \mathrm{msec}$. This is likely due to either spontaneous variability or decremental conduction properties of the bypass tract unmasked by the preceding long-short sequence, as evidenced by the shorter VA conduction time.

that point, orthodromic reciprocating tachycardia using a concealed accessory pathway was felt to be the most likely mechanism of the tachycardia, but this possibility became untenable when there was continuation of the tachycardia despite the occurrence of AV block. This continuation of the tachycardia despite AV nodal block, along with the other electrophysiologic findings in this case, can be explained by a reentrant tachycardia in which the anterograde limb was the AV node and the retrograde limb was a concealed atrionodal pathway.

\section{Prior Studies}

In an anatomic study by James, ${ }^{1}$ atrionodal pathways consisting of myofibrils and Purkinje fibers were found ubiquitously in an unselected population of patients. These fibers uniformly originated in the atrial septum. Although their potential function as a substrate for arrhythmias was commented upon, the electrophysiologic properties of these atrionodal pathways were not studied. In another anatomic study, Brechenmacher ${ }^{2}$ documented the existence of atrio-Hisian fibers in two patients who had a history of paroxysmal tachycardia. Again, both of these bypass tracts originated in the interatrial septum.
In contrast to these prior anatomic studies, the atrial insertion of the atrionodal pathway described in this case report was not in the atrial septum, but instead at the posterolateral tricuspid annulus. This suggests that the pathway coursed along the tricuspid annulus and was electrically insulated from the adjacent atrial myocardium. To our knowledge, this is the first report of an atrionodal nodal pathway either inserting outside of the septum or participating in a reentrant tachycardia.

\section{Conclusions}

This case is unique in that it represents the first report of a tachycardia that used a concealed atrionodal pathway as its retrograde limb. Of note is that if the single, short episode of spontaneous AV block had not occurred during the course of electrophysiologic testing, the tachycardia might have been mistaken for orthodromic reciprocating tachycardia using a concealed right free-wall accessory pathway. As noted, a ventricular extrastimulus introduced at the right ventricular apex coincident with His-bundle refractoriness failed to preexcite the atrium during tachycardia. Although this response does not exclude the possibility of orthodromic reciprocating tachycardia us- 


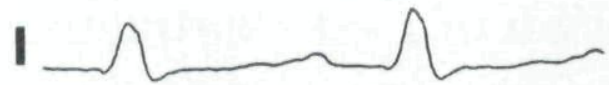

II

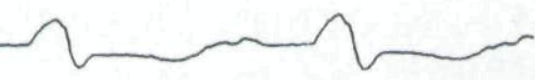

III
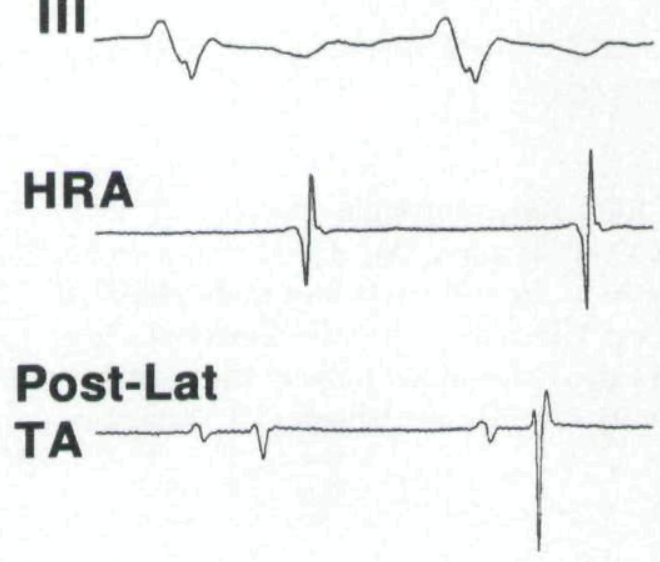

V1

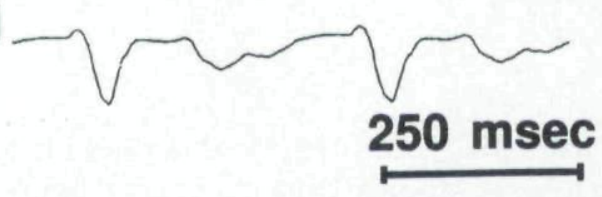

Figure 5. Intracardiac recording at the posterolateral tricuspid annulus (Post-Lat TA), which was the site of earliest atrial activation during the tachycardia. A single application of radiofrequency energy at this site terminated the tachycardia and eliminated VA conduction. HRA = high right atrium. ing an accessory pathway, it makes an ordinary right-sided accessory AV connection highly unlikely. ${ }^{4}$ This case suggests that concealed atrionodal pathways should be considered in the differential diagnosis when a ventricular extrastimulus coincident with His-bundle refractoriness does not preexcite the atrium during a presumed orthodromic reciprocating tachycardia.

\section{References}

1. James TN: Morphology of the human AV node, with remarks pertinent to its electrophysiology. Am Heart J 1961:62:756-771

2. Brechenmacher C: Atrio-His bundle tracts. Br Heart J 1975;37:853-855

3. Lev M, Leffler WB, Langendorf R, et al: Anatomic findings in a case of ventricular preexcitation (WPW) terminating in complete atrioventricular block. Circulation 1966;34:718-733.

4. Benditt DG, Benson DW, Dunnigan A, et al: Role of extrastimulus site and tachycardia cycle length in inducibility of atrial preexcitation by premature ventricular stimulation during reciprocating tachycardia. Am J Cardiol 1987;60:811-819. 
This document is a scanned copy of a printed document. No warranty is given about the accuracy of the copy. Users should refer to the original published version of the material. 